\title{
Aviso de valores de alerta por parte del laboratorio clínico en una red de salud universitaria
}

\author{
Ana María G uzmán $D^{1}$, Sandra Solari $S^{1}$, Marcela Lagos $L^{1}$, \\ Helena Poggi $M^{2 b}$, Tomás Sánchez $P^{2 a}$, Angélica Madrid Q 2a, \\ Jacqueline Parada B2a, Juan Carlos Román G 2a, \\ Luis Rodríguez $\mathbf{P}^{1}$, Teresa Q uiroga $\mathbf{G}^{1}$. \\ Laboratory alert value reporting \\ by the clinical laboratory \\ at an academic medical network
}

\begin{abstract}
Background: An alert value is a result suggesting that the patient is at imminent danger unless appropriate remedial actions begin promptly. Report of alert values (AV) by the clinical laboratories has taken special relevance in recent years due to its contribution to patient's care. Aim: To report results of AV informed during 2007 within the Health Network of the Pontificia Universidad Católica de Chile. Material and methods: Analysis of AV recorded in a centralized database of the laboratories of the health network, between January and December, 2007. Results: Total number of AV was 5.366, which represented $0.3 \%$ of total examinations and corresponded mainly to the clinical chemistry area. Potassium levels generated the higher number of AV detected, followed by positive blood cultures. Eighty two percent of AV corresponded to hospitalized patients. The greater number of $\mathrm{AV}$ was reported to intermediate and intensive care services. Thirty two percent of AV was informed to the physician or professional in charge of the patient within 5 minutes of obtaining the results and $79 \%$ within 30 minutes. Conclusions: To obtain a real impact on patient management, it is fundamental to shorten the lapse between the obtainment of tests results and the warning, supported on appropriate computerized systems, and to spread the procedure to all personnel involved in patient's care (Rev Méd Chile 2009; 137: 1137-44).
\end{abstract}

(Key w ords: Clinical laboratory information systems; Critical care; Online systems)

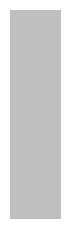

Recibido el 14 de enero, 2009. Aceptado el 23 de julio, 2009.

${ }^{1}$ Departamento de Laboratorios Clínicos. Facultad de Medicina, Pontificia Universidad Católica de Chile. Santiago de Chile. ${ }^{2}$ Servicio de Laboratorios Clínicos, Red de Salud, Pontificia Universidad Católica de Chile. Santiago de Chile.

aTecnólogo Médico

${ }^{b}$ Bioquímico

\footnotetext{
Correspondencia a: Dra. Ana María Guzmán D. Laboratorio de Urgencia, Hospital Clínico Pontificia Universidad Católica de Chile. Marcoleta 367, Santiago, Chile. Teléfono: 3543288. Fax: 3543489 - 3548571. E mail: amguzman@med.puc.cl
} 
U n "valor de alerta" (VA) o crítico se define como un resultado de laboratorio tan alejado de lo normal, que constituye una amenaza para la vida, a menos que se inicien acciones correctivas inmediatas. El concepto fue acuñado en la década de 1970 por Lundberg ${ }^{1}$, quien identificó la importancia del reconocimiento de estos VA por parte del personal del laboratorio y de su aviso oportuno al médico tratante. Luego de esta primera publicación, este concepto fue adoptado primero en Estados Unidos de Norteamérica (EE.UU.) como un requisito por parte del Clinical Laboratory Improvement Amendments (CLIA '88) ${ }^{2}$ y posteriormente por parte de diferentes entidades como el College of American Pathologists (CAP) ${ }^{3}$ y la Joint Commission on Accreditation on Healthcare Organizations $(\mathrm{JCAHO})^{4}$. De hecho, este tema ha adquirido especial relevancia, después de que esta última entidad divulgara las National Patient Safety Goals el año $2006^{5}$, en que no sólo hace de este procedimiento un deber para los laboratorios clínicos, sino que también, lo extiende a otras áreas de diagnóstico como radiología, electrocardiografía, etc., exigiendo además que el proceso sea monitorizado para demostrar mejoras en la rapidez del aviso.

En Chile, el Instituto Nacional de Normalización (INN) acredita a los laboratorios clínicos bajo la Norma Chilena 2547 "Laboratorios Clínicos Requisitos particulares para la calidad y competencia" que corresponde a la homologación de la Norma ISO $15189^{6}$. Esta norma también incorpora el procedimiento de aviso de VA, como parte de los requisitos técnicos en el punto 5.8 referido a Informe de Resultados. El año 2003 nuestro laboratorio comenzó a trabajar para la acreditación por el INN e introdujo el aviso de VA a sus actividades habituales, definiendo un procedimiento y elaborando el registro correspondiente.

El objetivo de este trabajo es analizar retrospectivamente los VA detectados durante el año 2007 por el Servicio de Laboratorios Clínicos de la Facultad de Medicina de la Pontificia Universidad Católica de Chile.

\section{MATERIALES Y MÉTODO}

Red de Salud UC. La Red comprende 3 centros de salud de nivel terciario (Hospital Clínico Universi- dad Católica con 391 camas, aprox. 106.669 días/ cama, Clínica UC con 106 camas/ aprox. 23.972 días/cama y Clínica UC San Carlos de Apoquindo con 105 camas/ aprox. 15.158 días/cama), 6 centros médicos para atención de pacientes ambulatorios y 12 Unidades de Toma de Muestras (UTMs) ubicadas en distintos puntos de la ciudad de Santiago de Chile, las cuales atienden alrededor de 384.000 pacientes ambulatorios al año.

El Servicio de Laboratorios Clínicos de la Pontificia Universidad Católica de Chile (SLC-PUC) atiende las necesidades de exámenes de esta Red, tanto de los pacientes ambulatorios como hospitalizados, a través de tres Laboratorios:

a) Laboratorio Centro Médico San Joaquín, al cual pertenecen 3 Unidades: Bioquímica, Microbiología y Biología Molecular/Citogenética.

b) Laboratorio de Urgencia Hospital Clínico UC.

c) Laboratorio de Urgencia Clínica UC San Carlos de Apoquindo.

Procedimiento de aviso de VA. En el procedimiento del SLC-PUC, se definen en un listado los analitos y resultados que constituyen VA, los cuales fueron extraídos de la literatura y consensuados con los médicos jefes de servicio ${ }^{7-10}$. Además, el listado incorpora por razones epidemiológicas, las baciloscopias positivas de muestras respiratorias, ya que tratándose de pacientes bacilíferos, se requiere indicación de aislamiento respiratorio inmediato. El listado utilizado el año 2007, se muestra en la Tabla 1.

El procedimiento estipula que cada vez que el profesional del laboratorio detecta un valor de alerta debe verificar en los algoritmos de decisión disponibles, si corresponde o no avisar (ver ejemplos en Figura 1). Si corresponde avisar, el profesional debe en primera instancia, contactar telefónicamente al médico tratante, ya sea de paciente hospitalizado o ambulatorio. En pacientes hospitalizados también existe la alternativa de informar el VA a la enfermera responsable del paciente, quien a su vez ubica al médico tratante. En pacientes ambulatorios, en cuyos casos se han agotado todas las instancias de contactar al médico tratante, se llama directamente al paciente o familiar y se le indica que retire el examen y que contacte a su médico a la brevedad.

En el procedimiento está definido que todo VA debe ser avisado, aun cuando el tiempo se extienda en forma exagerada o se deba recurrir al paciente para lograr ubicar al médico. 
Tabla 1. Listado de valores de alerta SLC-PUC año 2007

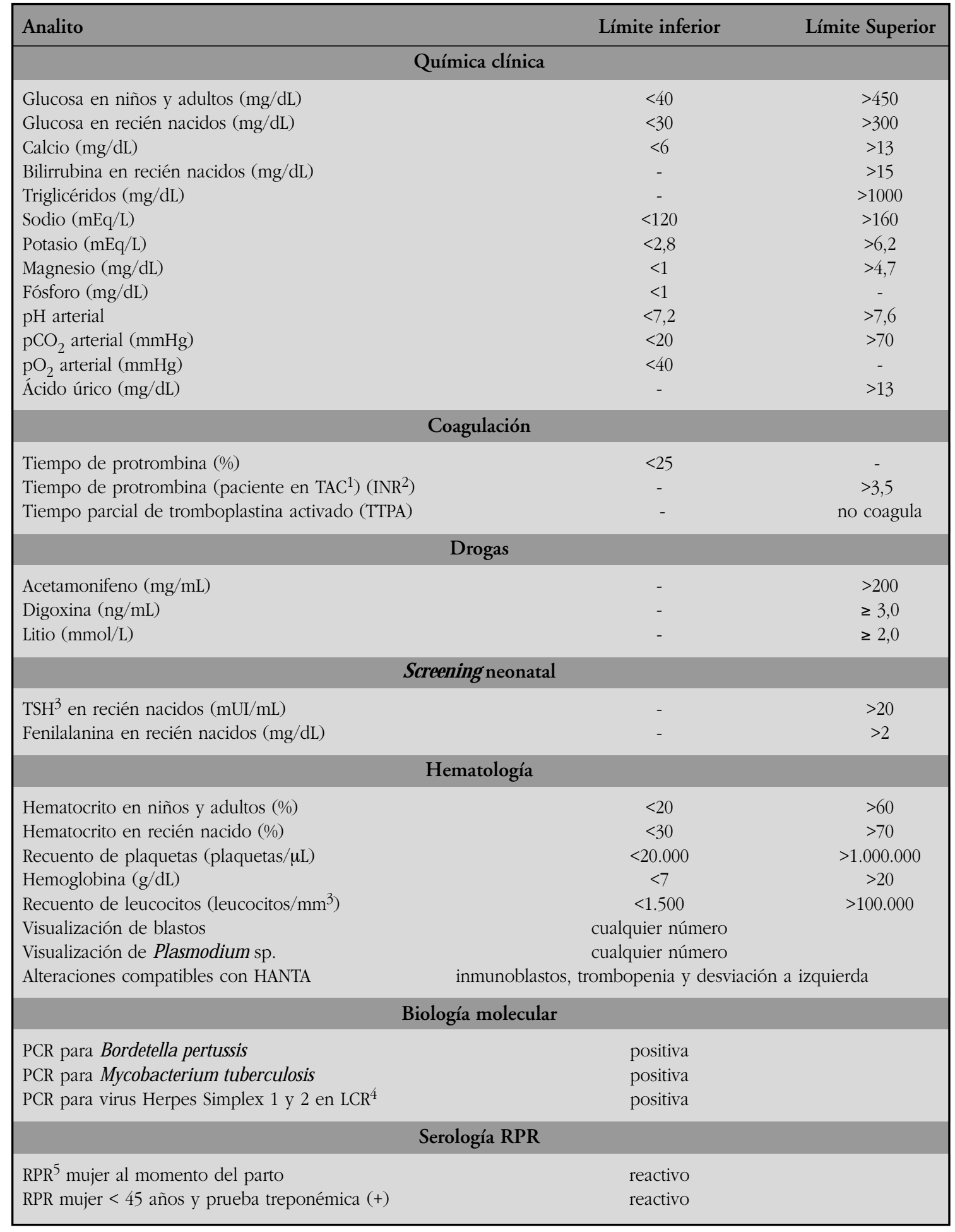




\begin{tabular}{|c|c|}
\hline \multicolumn{2}{|l|}{ Análisis de orina } \\
\hline $\begin{array}{l}\text { Glucosuria } \\
\text { Cetonuria }\end{array}$ & $\begin{array}{l}++++ \\
++++\end{array}$ \\
\hline \multicolumn{2}{|l|}{ Microbiología } \\
\hline $\begin{array}{l}\text { Baciloscopia en muestras respiratorias } \\
\text { Tinción de blanco calcofluor líquidos/tejido cavidades estériles } \\
\text { Tinción de Gram en líquido cefalorraquídeo } \\
\text { Cultivo de líquido cefalorraquídeo (si tinción Gram previa negativa) } \\
\text { Cultivo de Listeria sp. } \\
\text { Coprocultivo de E. coli O: } 157 \text { o Vibrio cholerae } \\
\text { Cultivo de Neisseria meningitidis (cualquier localización) } \\
\text { Cultivo de Bordetella, Difteria o Brucella sp. } \\
\text { Cultivo de Mycobacterium sp. (si baciloscopia previa negativa) } \\
\text { Cultivo de Bacillus anthracis, Yersinia pestis } \\
\text { Cultivo de Neisseria gonorrhoeae }<15 \text { años } \\
\text { Cultivo de Hemophilus influenzae }<15 \text { años } \\
\text { Serología de Chlamydia trachomatis }<15 \text { años } \\
\text { Serología de Leptospira sp. } \\
\text { Serología de Legionella sp. } \\
\text { Serología de Taenia solium } \\
\text { Observación microscópica búsqueda Plasmodium sp. }\end{array}$ & $\begin{array}{l}\text { positiva } \\
\text { positiva } \\
\text { positiva } \\
\text { positiva } \\
\text { positivo } \\
\text { positivo } \\
\text { positivo } \\
\text { positivo } \\
\text { positivo } \\
\text { positivo } \\
\text { positivo } \\
\text { positivo } \\
\text { positiva } \\
\text { positiva } \\
\text { positiva } \\
\text { positiva } \\
\text { positiva }\end{array}$ \\
\hline
\end{tabular}

${ }^{1}$ TAC: tratamiento anticoagulante, ${ }^{2}$ INR: International Normalized Ratio, ${ }^{3}$ TSH: hormona tiro-estimulante, ${ }^{4}$ LCR: líquido cefalorraquídeo, ${ }^{5} \mathrm{RPR}$ : reagina plasmática rápida.

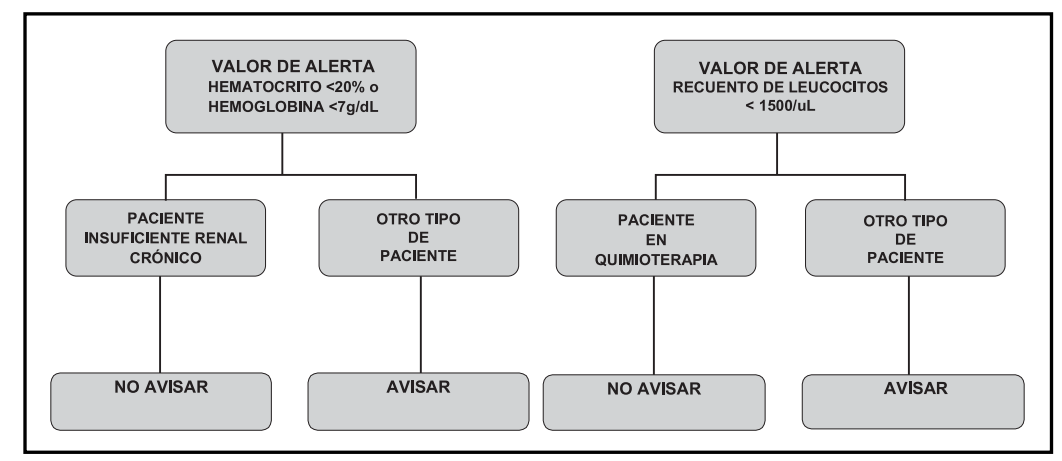

Figura 1. Ejemplos de algoritmos que ayudan al profesional del laboratorio a decidir el aviso de un valor de alerta.

Una vez que se contacta al médico tratante o enfermera, se le solicita que escriba el valor que se le está dictando y que lea el dato anotado (read-back) para asegurarse que el valor ha sido entendido y registrado correctamente. Además el profesional del laboratorio debe indicar que el examen se encuentra disponible en el sistema informático del laboratorio para su impresión.

El profesional responsable del laboratorio debe dejar registrados en una base de datos
(Microsoft ${ }^{\circledR}$ Access/Excel, Microsoft, Redmond, WA) todos los antecedentes relacionados al valor de alerta: nombre del paciente, edad, analito asociado, hora, fecha y responsables de la detección, aviso y recepción del VA.

Obtención de los datos. Para el análisis presentado en este trabajo se utilizaron los antecedentes de VA ingresados en la base de datos entre enero y diciembre de 2007. El número y tipo de exámenes realizados por 
los laboratorios en el mismo período fue extraído del sistema informático del laboratorio (SIL).

\section{Resultados}

Durante el año 2007, en el SLC-PUC se procesaron 1.895.043 exámenes. Su distribución según origen ambulatorio u hospitalizado y por área de análisis, se muestra en la Tabla 2. Del total de exámenes, 435.321 (23\%) correspondieron a exámenes de pacientes hospitalizados y 1.459 .722 (77\%) a exámenes de pacientes ambulatorios. Aproximadamente 60\% de los exámenes realizados correspondieron al área de química clínica-hormonas, 10\% al área de hematología y $10 \%$ al área de microbiología, distribución que se mantuvo tanto en exámenes provenientes de pacientes ambulatorios como hospitalizados.

En este período se registraron $5.366 \mathrm{VA}$, de los cuales la mayor parte fueron generados por exámenes correspondientes al área de química clínica (34\%), seguida por el área de hematología (27\%). Sin embargo, proporcionalmente al número de exámenes realizados en el período, el área en que más VA se generaron fue la microbiológica $(0,9 \%)$, seguida por el área de hematología (0,6\%). En el área de química clínica los VA detectados sólo representan el 0,2\% de su actividad (Tabla 3).

El $82,5 \%$ de los VA fueron detectados en el Laboratorio de Urgencia del Hospital Clínico (Figura 2). El número de VA detectados en este último, constituyeron el 1,1\% de su actividad en contraste con los otros Laboratorios de la Red, en que los VA representaron menos del $0,2 \%$ de los exámenes realizados (Tabla 4). El mayor número de VA correspondieron a pacientes hospitalizados en Servicios Intensivos e Intermedios del Hospital Clínico. A cada cama de estos servicios correspondieron alrededor de 22 VA anuales, comparado con los 4,7 y 3,5 VA por cama de salas y pensionados respectivamente, durante el mismo periodo (Tabla 5).

Si se desglosan los resultados según analito, se observa que los VA fueron generados mayoritariamente a partir de determinaciones de potasio (19\%) y de hemocultivos positivos (14\%) (Figura 3). Otros exámenes cuyas alteraciones generaron frecuentemente VA correspondieron al recuento de plaquetas (11\%), tiempo parcial de tromboplastina activado (TTPA) (10\%) y hematocrito (9\%).

En cuanto al tiempo transcurrido entre la detección del VA en el laboratorio y el aviso al médico tratante, destaca que $32,1 \%(\mathrm{n}=1724)$ de los VA se avisaron antes de los 5 minutos y 20,9\% ( $n=1124)$ se avisaron después de los 30 minutos (Figura 4).

\section{DisCUSIÓN}

El aviso de los VA, aunque descrito desde hace más de tres décadas, ha sido incorporado más bien recientemente a las prácticas habituales de los laboratorios clínicos y a pesar de ser actual-

Tabla 2. Número y porcentaje de exámenes provenientes de pacientes ambulatorios, hospitalizados y totales según área de análisis, realizados en el SLC-PUC

\begin{tabular}{|lrrrrrr|}
\hline Área de análisis & \multicolumn{2}{c}{$\begin{array}{c}\text { Exámenes pacientes } \\
\text { ambulatorios }\end{array}$} & \multicolumn{2}{c}{$\begin{array}{c}\text { Exámenes pacientes } \\
\text { hospitalizados }\end{array}$} & \multicolumn{2}{c|}{$\begin{array}{c}\text { Exámenes } \\
\text { totales }\end{array}$} \\
& \multicolumn{1}{c}{$\mathbf{n}$} & \multicolumn{1}{c}{$\%$} & \multicolumn{1}{c}{$\mathbf{n}$} & \multicolumn{1}{c}{$\%$} & $\mathbf{n}$ & $\%$ \\
\hline Química-hormonas & 970.315 & 66,5 & 257.645 & 59,2 & 1.227 .960 & 64,8 \\
Hematología & 180.106 & 12,3 & 58.269 & 13,4 & 238.375 & 12,6 \\
Coagulación & 80.723 & 5,5 & 54.720 & 12,6 & 135.443 & 7,1 \\
Análisis de orina & 90.500 & 6,2 & 7.956 & 1,8 & 98.456 & 5,2 \\
Microbiología & 105.408 & 7,2 & 42.142 & 9,7 & 147.550 & 7,8 \\
Biología molecular & 4.494 & 0,3 & 735 & 0,2 & 5.229 & 0,3 \\
Screening neonatal & 187 & 0,0 & 5.216 & 1,2 & 5.403 & 0,3 \\
Serología RPR & 10.370 & 0,7 & 3.773 & 0,9 & 14.143 & 0,7 \\
Drogas & 17.619 & 1,2 & 4.865 & 1,1 & 22.484 & 1,2 \\
Total & 1.459 .722 & 100,0 & 435.321 & 100,0 & 1.895 .043 & 100,0 \\
\hline
\end{tabular}

n: número, \%: porcentaje, RPR: prueba de reagina plasmática rápida 
Tabla 3. Número y porcentaje de VA según área de análisis y en relación al total de exámenes realizados

\begin{tabular}{|c|c|c|c|}
\hline \multirow[t]{2}{*}{ Area de análisis } & \multicolumn{2}{|c|}{ VA detectados } & \multirow{2}{*}{$\begin{array}{c}\text { VA/exámenes realizados } \\
\%^{*}\end{array}$} \\
\hline & $\mathbf{n}$ & $(\%)$ & \\
\hline Química-hormonas & 1.831 & 34,1 & 0,2 \\
\hline Hematología & 1.447 & 27,0 & 0,6 \\
\hline Coagulación & 738 & 13,8 & 0,5 \\
\hline Análisis de orina & 53 & 1,0 & 0,1 \\
\hline Microbiología & 1.263 & 23,5 & 0,9 \\
\hline Biología molecular & 28 & 0,5 & 0,5 \\
\hline Screening neonatal & 3 & 0,1 & 0,1 \\
\hline Serología RPR & 3 & 0,1 & 0,0 \\
\hline Drogas & 0 & 0,0 & 0,0 \\
\hline Total & 5.366 & 100,0 & 0,3 \\
\hline
\end{tabular}

*Porcentaje respecto del total de exámenes realizados (ver Tabla 2).

n: número, \%: porcentaje, VA: valores de alerta, RPR: reagina plasmática rápida.

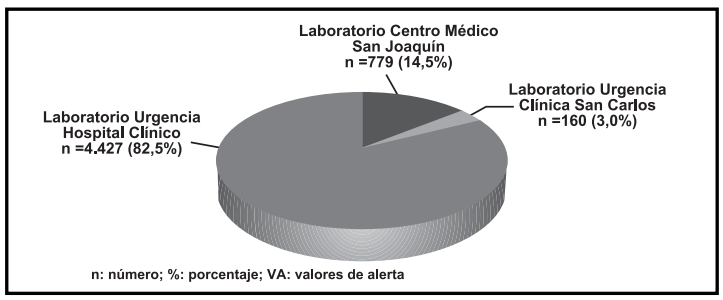

Figura 2. VA detectados en el SLC-PUC según laboratorio que detecta.

Tabla 4. Proporción de VA respecto del número total de exámenes realizados por laboratorio

\begin{tabular}{|c|c|c|c|}
\hline Laboratorio & $\begin{array}{l}\text { VA detectados } \\
\mathbf{n}\end{array}$ & $\begin{array}{c}\text { Exámenes realizados } \\
\mathbf{n}\end{array}$ & $\begin{array}{c}\text { VA/exámenes realizados } \\
\%\end{array}$ \\
\hline Centro Médico San Joaquín & 779 & 1.405 .982 & 0,1 \\
\hline Urgencia Clínica UC San Carlos & 160 & 90.742 & 0,2 \\
\hline Urgencia Hospital Clínico & 4.427 & 398.319 & 1,1 \\
\hline Total & 5.366 & 1.895 .043 & 0,3 \\
\hline
\end{tabular}

n: número, \%: porcentaje, VA: valores de alerta

Tabla 5. VA correspondientes a pacientes hospitalizados en el Hospital Clínico UC, clasificados según complejidad del servicio clínico

\begin{tabular}{|lrrr|}
\hline Tipo servicio & VA (n) & Camas (n) & VA/cama \\
\hline Intensivos & 1.158 & 52 & 22,3 \\
Intermedios & 597 & 27 & 22,1 \\
Salas & 890 & 191 & 4,7 \\
Pensionado & 403 & 116 & 3,5 \\
Total & 3.048 & 386 & 7,9 \\
\hline
\end{tabular}

n: número, VA: valores de alerta 


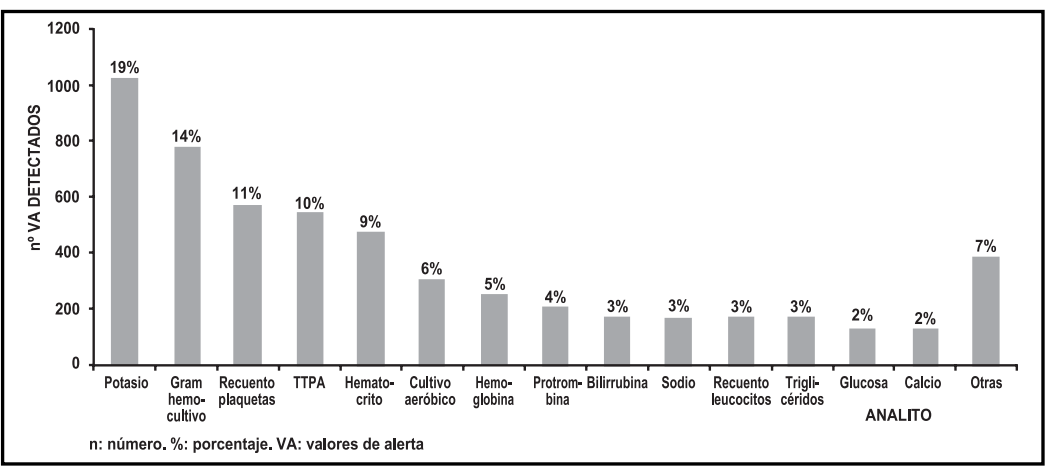

Figura 3. VA detectados por el SLC-PUC, según analito.

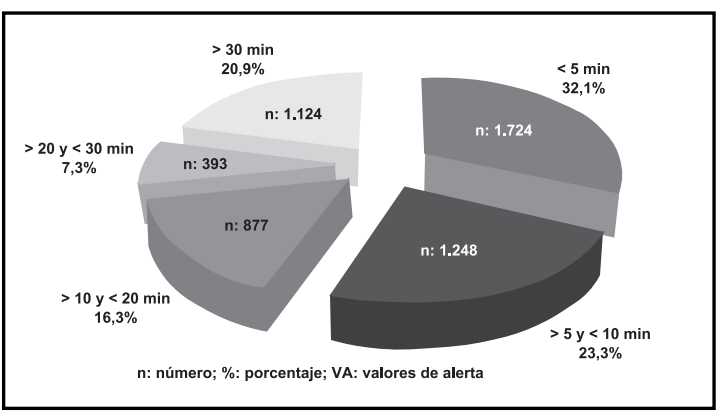

Figura 4. Tiempo transcurrido entre detección del VA y aviso al médico o profesional responsable del paciente.

mente un requisito normativo, la bibliografía es escasa. Este trabajo, de corte descriptivo, tiene como propósito dar a conocer cómo se está cumpliendo el procedimiento en nuestra red de salud, y detectar posibles áreas de mejora.

Procedimiento de valores de alerta. Si bien no existe un procedimiento único y éste debe ser adecuado a cada realidad, existen algunas recomendaciones en la literatura $^{11}$ que el SLC-PUC ha ido incorporando. Entre ellas nos parece importante destacar: los límites que definen los VA deben ser respetados estrictamente, el aviso de VA debe realizarse una vez confirmado el resultado, la responsabilidad del aviso y de la recepción debe ser asignada a personal profesional y además, debe llevarse un registro completo del proceso. A esto se agrega la implementación de la práctica del "read-back" (secuencia de anotar lo dictado y leer lo anotado) ya comentada, la cual es una meta de seguridad estipulada por Joint Commission, que tiene como objetivo evitar errores en las comunicaciones verbales dentro de una institución de salud ${ }^{5}$.

El listado de VA de un centro de salud debe ser adecuado al nivel de complejidad y al tipo de exámenes que en él se realizan, y revisado en forma periódica con los médicos de los servicios clínicos, de manera de incorporar o retirar analitos, y de cambiar los límites que definen valores de corte cuando corresponda. Para evitar el aviso excesivo de VA, es importante acotar el listado a los analitos de real impacto en el cuidado del enfermo y poner algoritmos de decisión a disposición del profesional del laboratorio clínico. De acuerdo a una encuesta reciente del College of American Pathologists ${ }^{11}$, la mayor parte de los VA de nuestro listado (Tabla 1) están incluidos también en los listados de instituciones de salud similares.

Número y distribución de VA. De acuerdo a lo publicado, la proporción de VA detectados en relación al número total de exámenes realizados es variable, dependiendo de la complejidad del centro de salud y los pacientes que allí se atienden $(0,05 \text { a } 1 \%)^{9}$. La proporción observada en el SLC-PUC (0,3\%) concuerda con lo descrito en un centro similar ${ }^{12}$, siendo mayor en el Laboratorio de Urgencia del Hospital Clínico (1,1\%) y específicamente en los Servicios de Cuidados Intensivos e Intermedios, donde se encuentran los pacientes de mayor complejidad.

Los analitos más frecuentemente avisados corresponden a lo descrito en la literatura (potasio, recuento de plaquetas, TTPA y hematocrito) ${ }^{12}$, exceptuando los hemocultivos positivos, para los cuales no encontramos estudios con los cuales comparar nuestros datos.

Tiempo transcurrido entre la detección del VA en el laboratorio y el aviso al médico tratante. El aviso del VA debe ser rápido y oportuno para el cuidado del enfermo; VA avisados muy tardíamente tendrán bajo impacto y serán poco costo/ efectivos para la institución ${ }^{11}$. 
A pesar de que en nuestra red de salud la mayor parte de los VA (79\%) se avisaron antes de 30 minutos, 21\% de ellos se avisaron en tiempos mayores. En la literatura existe gran dispersión en los tiempos de aviso de $\mathrm{VA}^{9-12}$, lo cual se debe fundamentalmente a si se dispone o no de tecnologías de apoyo para ubicar al profesional responsable. El principal problema en nuestra Red es que no disponemos de una vía rápida o exclusiva de comunicación con los servicios clínicos, ni tampoco de un sistema informático que permita dar este tipo de aviso al servicio clínico correspondiente. Paralelamente a estos sistemas de apoyo, es necesario contar con una base de datos actualizada de los médicos tratantes de la Red, sus buscapersonas o celulares. Una dificultad adicional la representan pacientes ambulatorios que son atendidos en consultas cuyos teléfonos no están disponibles las 24 horas $^{12}$ o en que la orden médica no incluye datos del médico tratante o éstos son incompletos o antiguos.

Desafíos futuros y posibles mejoras. De acuerdo a nuestros resultados, una de las principales tareas que se nos plantea, es la necesidad de acortar los tiempos de aviso del VA. Para ello debemos difundir nuevamente el procedimiento de VA a todo el personal dentro de nuestra Institución, especialmente al servi-

\section{REFERENCIAS}

1. LundBerg GD. When to panic over abnormal values. MLO Med Lab Observ 1972; 4: 47-54.

2. LundBerg GD. Panic values - five years later. MLO Med Lab Observ 1977; 9: 27-34.

3. Center for Medicare and Medicaid Services. Department of health and human Services Clinical. Laboratory Improvement Amendments of 1988: 68 Federal Register 1047 (2003) (Codified at 42 CFR 493.1291 (g)).

4. Rabinovitch A. The College of American Pathologists (CAP) Laboratory Accreditation Checklists. Northfiled III: College of American Pathologists; 2000.

5. Joint Commission on the Accreditation of Healthcare Organizations. National Patient Safety Goals. Available at: htpp:/www.jcaho.org/accredited organizations/patient safety/npsg.htm.

6. Norma Chilena Oficial NCh 2547 Of. 2003 Laboratorios Clínicos - Requisitos Particulares para la Calidad y Competencia. Instituto Nacional de Normalización.

7. Kost GJ. Critical limits for urgent clinician notification at US medical centers. JAMA 1990; 263: 704-7.

8. Kost GJ. Critical limits for emergency clinician cio de enfermería del hospital. También debe educarse al personal de cajas que atiende a nuestros pacientes ambulatorios, haciendo hincapié en la importancia de actualizar los datos del médico en el sistema informático, que es el punto de partida para cualquier aviso de VA de un paciente ambulatorio.

En forma paralela a una mayor difusión, el tiempo transcurrido entre la detección y el aviso de los VA debe seguir monitorizándose en forma continua, por lo cual este parámetro se ha incorporado a nuestros indicadores de calidad, estableciéndose una meta de aviso menor a 30 minutos para más del 90\% de los VA detectados.

Una vez que se ha implementado el procedimiento de aviso de VA en el laboratorio, es necesario evaluar el tiempo que efectivamente el médico tratante se demora en recibir la información, revisar los antecedentes del paciente e iniciar el tratamiento o la acción correctiva correspondiente. En trabajos donde esto ha sido evaluado, la indicación de tratamiento demora alrededor de 2,5 horas $^{13,14}$, cifra que indudablemente puede mejorar. Si bien el aviso de VA por parte del laboratorio es un aporte innegable al manejo del paciente, éste debe necesariamente integrarse con acciones inmediatas en los servicios clínicos, para que tenga un impacto en el cuidado del enfermo.

notification at United States children's hospitals. Pediatrics 1991; 88: 597-603.

9. Howanitz PJ, Steindel SJ, Heard NV. Laboratory critical values policies and procedures: a College of American Pathologists Q-Probes Study in 623 Institutions. Arch Pathol Lab Med 2002; 126: 663-9.

10. Wagar E, Friedber R, Souers R, Stankovic A. Critical values comparison: a College of American Pathologists Q-Probes Survey of 163 Clinical Laboratories. Arch Pathol Lab Med 2007; 131: 1769-75.

11. Emancipator K. Critical values ASP practice parameter. Am J Clin Pathol 1997; 108: 247-53.

12. Dighe A, Rao A, Conkley A, Lewandrowski K. Analysis of laboratory critical value reporting at a large academic medical center. Am J Clin Pathol 2006; 125: 758-64.

13 Kuperman G, Boyle D, Jha A, Rittenberg MA, Ma'luf N, TANaSijevic M ET AL. How promptly are inpatients treated for critical laboratory results? JAMA 1998; 5: 112-9.

14.Valenstein P, Wagar E, Stankovic A, Walsh M, SchneiDER F. Notification of critical results: a College of American Pathologists Q-probes study of 121 institutions. Arch Pathol Lab Med 2008; 132: 1862-7. 